\title{
Modified generalized linear failure rate distribution: Properties and reliability analysis
}

\author{
Ezzatallah Baloui Jamkhaneh*
}

Department of Statistics, Qaemshahr Branch, Islamic Azad University, Qaemshahr, Iran

\begin{tabular}{l}
\hline C H R O N I C L E \\
\hline Article history: \\
Received September 222013 \\
Received in Revised Format \\
April 62014 \\
Accepted April 122014 \\
Available online \\
April 18 2014 \\
\hline Keywords: \\
Fuzzy reliability \\
Fuzzy MGLFR distribution \\
Hazard function
\end{tabular}
A B S T R A C T

\begin{abstract}
This paper introduces a new comprehensive four-parameter distribution called the modified generalized linear failure rate (MGLFR) distribution. The method generalizes some well-known and most commonly used distributions in reliability such as exponential, Rayleigh, linear failure rate, generalized linear failure rate and modified Weibull distribution. The study also investigates some essential properties of this new distribution and considers the problem of the evaluation of system reliability by describing the lifetimes of components based on a fuzzy MGLFR distribution and by developing fuzzy reliability characteristics. The results can be applied to determine the reliability of real objects where parameters of lifetime variable are subject to uncertainty.
\end{abstract}

\section{Introduction}

There are literally numerous distributions for modeling lifetime data such as exponential, Weibull, Rayleigh, linear failure rate or generalized exponential distributions. Reliability has always been a key role in designing engineering systems. The most frequently used functions in lifetime data analysis and reliability engineering are the reliability function (survival function), and hazard function. Survival function gives the probability of an item operating for a certain amount of time without failure. Exponential distribution maintains only constant hazard function whereas Rayleigh, linear failure rate and generalized exponential distribution may have only monotone hazard functions. The Weibull distribution function was introduced by Fisher and Tippett in 1928. The Swedish physicist Wallodi Weibull (1939) used this probability distribution for describing the lifetime of components with variable failure rate. Burr (1942) introduced twelve different forms of cumulative distribution functions for modeling lifetime data. Among those distributions, Burr Type X and Burr Type XII are the most popular ones.

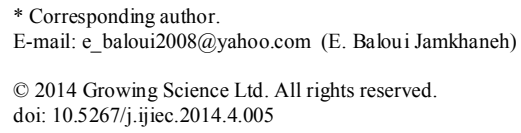


Surles and Padgett (2001) introduced two-parameter Burr Type X distribution and correctly named as the generalized Rayleigh (GR) distribution. They showed that the GR distribution could be used quite effectively in modeling strength data and in modeling general lifetime data. Mudholkar and Srivastava (1993) introduced the exponential Weibull family, which contains important physical interpretation. Gupta and Kundu (1999a) introduced generalized exponential distribution (GED). The two-parameter GED can have non-increasing and non-decreasing failure rates depending on the shape parameter. Sarhan and Zaindin (2009) introduced a new three-parameter distribution called the Modified Weibull distribution (MWD). This distribution generalizes the well-known (1) exponential distribution, (2) linear failure rate distribution, (3) generalized exponential distribution, and (4) generalized Rayleigh distribution. The MWD can be used to describe several reliability models. Sarhan and Kundu (2009) introduced generalized linear failure rate distribution. It can have increasing, decreasing and bathtub shaped hazard functions.

There are several methods and models in classical reliability theory, which assume that all parameters of lifetime density functions are precise. However, in the real world applications, randomness and fuzziness are often mixed up in the lifetimes of systems. However, the parameters sometimes cannot record precisely due to machine errors, experiment, personal judgment, estimation or some other unexpected situations. When parameter in the lifetime distribution is fuzzy, the conventional reliability system may have difficulty for handling reliability and hazard functions. The theory of fuzzy reliability was proposed and development by several authors (Cai et al., 1991, 1993; Cai, 1996; Chen \& Mon, 1993; Hammer, 2001; Onisawa \& Kacprzyk, 1995; Utkin \& Gurov, 1995).

Aliev and Kara (2004) considered fuzzy system reliability analysis using time dependent fuzzy set and the concept of alpha-cut. Utkin (1994) discussed imprecise reliability models for the general lifetime distribution classes. He applied the theory of imprecise probability to reliability analysis. $\mathrm{Wu}(2006)$ considered fuzzy Bayesian system reliability assessment based on exponential distribution. Guo et al. (2007) proposed a credibility hazard concept associated with fuzzy lifetimes. Guo et al. (2007) considered random fuzzy variable modeling on repairable system. Yao et al. (2008) applied a statistical methodology in fuzzy system reliability analysis and provided a fuzzy estimation of reliability. Karpisek et al. (2010) described two fuzzy reliability models based on the Weibull fuzzy distribution. Baloui Jamkhaneh and Nozari (2012) investigated fuzzy system reliability analysis based on confidence interval. Garg et al. (2013) considered reliability analysis of the engineering systems using intuitionistic fuzzy set theory. Pak et al. (2013) presented a Bayesian approach to estimate the parameter and reliability function of Rayleigh distribution from fuzzy lifetime data. Baloui Jamkhaneh $(2011,2014)$ evaluated reliability function using fuzzy lifetime distribution.

The aim of this paper is to introduce the modified generalizes linear failure rate distribution (MGLFRD). We consider the problem of the evaluation of system reliability, in which the lifetimes of components are described using MGLFR distribution with fuzzy parameter.

\section{Modified generalized linear failure rate distribution}

In this section, we introduce a new four-parameter distribution function called as modified generalized linear failure rate distribution with four parameters $a, b, c, \theta$ denoted as $\operatorname{MGLFRD}(a, b, c, \theta)$. Lifetime random variable of $X$ with MGLFR distribution has probability density function as follows

$$
\begin{gathered}
f(x, a, b, c, \theta)=\theta\left(a+b c x^{c-1}\right)\left[1-\exp \left(-\left(a x+b x^{c}\right)\right)\right]^{\theta-1} \exp \left(-\left(a x+b x^{c}\right)\right), \\
x>0, a \geq 0, b \geq 0, c \geq 0, \theta>0
\end{gathered}
$$

and its distribution function is as follows,

$$
F(t, a, b, c, \theta)=\left[1-\exp \left(-\left(a t+b t^{c}\right)\right)\right]^{\theta}, t>0, a \geq 0, b \geq 0, c \geq 0, \theta>0 .
$$


It is immediate that from $\operatorname{MGLFRD}(a, b, c, \theta)$, the following special cases can be derived:

a) Linear failure distribution $\operatorname{LFRD}(a, b)$, when $\theta=1, c=2$

$f(x)=(a+2 b x) \exp \left(-\left(a x+b x^{2}\right)\right), x>0, a \geq 0, b \geq 0$.

b) Generalized exponential distribution $G E(a, \theta)$, when $b=0$,

$f(x)=\theta a[1-\exp (-(a x))]^{\theta-1} \exp (-(a x)), x>0, a \geq 0, c \geq 0, \theta>0$.

c) Generalized Rayleigh distribution $G R D(b, \theta)$, when $a=0, c=2$.

$f(x)=2 \theta b x\left[1-\exp \left(-\left(b x^{2}\right)\right)\right]^{\theta-1} \exp \left(-\left(b x^{2}\right)\right), x>0, b \geq 0, \theta>0$.

d) Exponential Weibull distribution $E W(b, c, \theta)$, when $a=0$,

$f(x)=\theta b c x^{c-1}\left[1-\exp \left(-b x^{c}\right)\right]^{\theta-1} \exp \left(-b x^{c}\right), x>0,, b \geq 0, c \geq 0, \theta>0$.

e) Weibull distribution $W(b, c)$, when $a=0, \theta=1$.

$f(x)=b c x^{c-1} \exp \left(-b x^{c}\right), x>0,, b>0, c>0$

f) Exponential distribution $E(a)$, when $\theta=1, b=0$,

$f(x)=a \exp (-a x), x>0, a>0$

g) Generalized Linear Failure Rate Distribution $\operatorname{GLFRD}(a, b, \theta)$, when $c=2$,

$f(x)=\theta(a+2 b x)\left[1-\exp \left(-\left(a x+b x^{2}\right)\right)\right]^{\theta-1} \exp \left(-\left(a x+b x^{2}\right)\right), x>0, a \geq 0, b \geq 0, \theta>0$.

h) Modified Weibull Distribution $\operatorname{MWD}(a, b, c)$, when $\theta=1$,

$f(x)=\left(a+b c x^{c-1}\right) \exp \left(-\left(a x+b x^{c}\right)\right), x>0, a \geq 0, b \geq 0, c \geq 0$.

\section{Statistical properties of MGLFRD}

\subsection{The quantile $q^{\text {th }}\left(x_{q}\right)$}

The quantile $q^{\text {th }}$ of the $\operatorname{MGLFRD}(a, b, c, \theta)$ is given by

$q=\int_{0}^{x_{q}} f(x, a, b, c, \theta) d x=F\left(x_{q}, a, b, c, \theta\right)$

So, the $x_{q}$ of $\operatorname{MGLFRD}(a, b, \theta)$ can be obtained with equation as follows, $b x_{q}^{c}+a x_{q}+\operatorname{Ln}\left(1-q^{\frac{1}{\theta}}\right)=0$. 
i) if $c=1$, then $x_{q}=\frac{-\operatorname{Ln}\left(1-q^{\frac{1}{\theta}}\right)}{a+b}$

ii) if $c=2$, then $x_{q}=\frac{-a+\sqrt{a^{2}-4 b \operatorname{Ln}\left(1-q^{\frac{1}{\theta}}\right)}}{2 b}$

iii) if $q=0.5$ in $\mathrm{i}$ and ii, then we will gain distribution median.

\subsection{Moments}

The following Lemma gives the $\mathrm{k}^{\text {th }}$ moment of $\operatorname{MGFRD}(a, b, c, \theta)$, when $\theta \geq 1$.

Lemma 1: If $\mathrm{X}$ has $M G L F R D(a, b, c, \theta)$, then the $\mathrm{k}^{\text {th }}$ moment of $\mathrm{X}\left(\mu^{(k)}\right)$, is given as follows:

for $a=0, b>0$ :

$\mu^{(k)}=\frac{\theta \Gamma\left(\frac{k}{c}+1\right)}{b^{\frac{k}{c}}} \sum_{i=0}^{\infty} \frac{\left(\begin{array}{c}\theta-1 \\ i\end{array}\right)(-1)^{i}}{(i+1)^{\frac{k}{c}+1}}$

for $a>0, b \geq 0$ :

$\mu^{(k)}=\sum_{i=0}^{\infty} \sum_{j=0}^{\infty} \theta\left(\begin{array}{l}\theta-1 \\ i\end{array}\right)(-1)^{i} \frac{w_{i}^{(j)}(0)}{j !}\left[\frac{a \Gamma(k+j+1)}{[a(i+1)]^{k+j+1}}+\frac{b c \Gamma(k+j+c)}{[a(i+1)]^{k+j+c}}\right]$

Proof: under definition of $\mathrm{k}^{\text {th }}$ moment, we will have

$\mu^{(k)}=\int_{0}^{\infty} x^{k} f(x, a, b, c, \theta) d x$,

then

$\mu^{(k)}=\int_{0}^{\infty} x^{k} \theta\left(a+b c x^{c-1}\right)\left[1-\exp \left(-\left(a x+b x^{c}\right)\right)\right]^{\theta-1} \exp \left(-\left(a x+b x^{c}\right)\right) d x$

since $0<\exp \left(-\left(a x+b x^{c}\right)<1, x>0\right.$, by using the binomial series expansion we have

$\left[1-\exp \left(-\left(a x+b x^{c}\right)\right)\right]^{\theta-1}=\sum_{i=0}^{\infty}\left(\begin{array}{l}\theta-1 \\ i\end{array}\right)(-1)^{i} \exp \left(-i\left(a x+b x^{c}\right)\right.$

then

$\mu^{(k)}=\theta \int_{0}^{\infty} x^{k}\left(a+b c x^{c-1}\right) \sum_{i=0}^{\infty}\left(\begin{array}{l}\theta-1 \\ i\end{array}\right)(-1)^{i} \exp \left(-(i+1)\left(a x+b x^{c}\right)\right) d x$ 
Since the inner quantity of the summation is computable, interchanging the integration and summation yields,

$$
\mu^{(k)}=\sum_{i=0}^{\infty}\left(\begin{array}{l}
\theta-1 \\
i
\end{array}\right)(-1)^{i} \theta \int_{0}^{\infty} x^{k}\left(a+b c x^{c-1}\right) \exp \left(-(i+1)\left(a x+b x^{c}\right)\right) d x
$$

i) if $a=0, b>0$ then

$$
\int_{0}^{\infty} x^{k}\left(a+b c x^{c-1}\right) \exp \left(-(i+1)\left(a x+b x^{c}\right)\right) d x=\int_{0}^{\infty} b c x^{k+c-1} \exp \left(-(i+1) b x^{c}\right) d x=\frac{b \Gamma\left(\frac{k}{c}+1\right)}{((i+1) b)^{\frac{k}{c}+1}}
$$

then

$$
\mu^{(k)}=\frac{\theta \Gamma\left(\frac{k}{c}+1\right)}{b^{\frac{k}{c}}} \sum_{i=0}^{\infty} \frac{\left(\begin{array}{c}
\theta-1 \\
i
\end{array}\right)(-1)^{i}}{(i+1)^{\frac{k}{c}+1}}
$$

ii) if $a>0, b \geq 0$, using the Taylor expansion of the function $\exp \left(-(i+1) b x^{c}\right)$ given by

$$
\exp \left(-(i+1) b x^{c}\right)=\sum_{j=0}^{\infty} \frac{w_{i}^{(j)}(0)}{j !} x^{j}, w_{i}^{(j)}(0)=\left.\frac{d^{j}}{d x^{j}} \exp \left(-(i+1) b x^{c}\right)\right|_{x=0}
$$

so

$$
\begin{aligned}
\mu^{(k)} & =\sum_{i=0}^{\infty} \sum_{j=0}^{\infty} \theta\left(\begin{array}{l}
\theta-1 \\
i
\end{array}\right)(-1)^{i} \frac{w_{i}^{(j)}(0)}{j !} \int_{0}^{\infty} x^{k+j}\left(a+b c x^{c-1}\right) \exp (-(i+1) a x) d x \\
& =\sum_{i=0}^{\infty} \sum_{j=0}^{\infty} \theta\left(\begin{array}{l}
\theta-1 \\
i
\end{array}\right)(-1)^{i} \frac{w_{i}^{(j)}(0)}{j !}\left[\frac{a \Gamma(k+j+1)}{[a(i+1)]^{k+j+1}}+\frac{b c \Gamma(k+j+c)}{[a(i+1)]^{k+j+c}}\right] .
\end{aligned}
$$

\subsection{Distribution of order statistics}

Let $X_{1}, X_{2}, \ldots, X_{n}$ be a random sample from $\operatorname{MGLRD}(a, b, c, \theta)$. Let $Y_{1} \leq Y_{2} \leq \ldots \leq Y_{n}$ denote the order statistics obtained from this sample. Then PDF of $Y_{i}$ is given by,

$$
\begin{aligned}
g_{i}(y) & =\frac{n !}{(i-1) !(n-i) !} f(y, a, b, c, \theta) F(y, a, b, c, \theta)^{i-1}(1-F(y, a, b, c, \theta))^{n-i} \\
& \left.=\frac{n !}{(i-1) !(n-i) !} f(y, a, b, c, \theta) \sum_{j=0}^{n-i}\left(\begin{array}{l}
n-i \\
j
\end{array}\right)(-1)^{j} F(y, a, b, c, \theta)\right)^{j+i-1} \\
& \left.=\sum_{j=0}^{n-i} \frac{n !}{(i-1) !(n-i) !} \times\left(\begin{array}{l}
n-i \\
j
\end{array}\right)(-1)^{j} \times \frac{f(y, a, b, c, \theta(i+j))}{i+j}=\sum_{j=0}^{n-i} k_{j}(n, i) f\left(y, a, b, c, \theta_{i+j}\right)\right)
\end{aligned}
$$

where

$$
k_{j}(n, i)=\frac{n(-1)^{j}\left(\begin{array}{l}
n-1 \\
i-1
\end{array}\right)\left(\begin{array}{l}
n-i \\
j
\end{array}\right)}{i+j}, \theta_{r}=r \theta
$$

Lemma 2: If $X_{1}, X_{2}, \ldots, X_{n}$ be a random sample from $\operatorname{MGLFRD}(a, b, c, \theta)$. Then $Y_{n}$ follows $\operatorname{MGLFRD}(a, b, c, n \theta)$. 
Remark: Consider a parallel system of $k$ identical and independent components, when each component has follows $M W D(a, b, c)$. In this case, the distribution function of a parallel system is as $\operatorname{MGLFRD}(a, b, c, k)$.

Lemma 3: Let $Y_{i}$ denote the $i^{\text {th }}$ order statistics, then the $k^{\text {th }}$ moment of $Y_{i}\left(\mu^{(k)}\right)$, is given as follows:

for $a=0, b>0$ :

$\mu^{(k)}=\frac{\Gamma\left(\frac{k}{c}+1\right)}{b^{\frac{k}{c}}} \sum_{j=0}^{n-i} \sum_{m=0}^{\infty} k_{j}(n, i) \frac{\left(\begin{array}{c}\theta_{i+j}-1 \\ m\end{array}\right)(-1)^{m} \theta_{i+j}}{(m+1)^{\frac{k}{c}+1}}$

for $a>0, b \geq 0$ :

$$
\mu^{(k)}=\sum_{m=0}^{\infty} \sum_{l=0}^{\infty} \sum_{j=0}^{n-i} \theta_{i+j} k_{j}(n, i)\left(\begin{array}{l}
\theta_{i+j}-1 \\
m
\end{array}\right)(-1)^{m} \frac{w_{m}^{(l)}(0)}{l !}\left[\frac{a \Gamma(k+l+1)}{[a(m+1)]^{k+l+1}}+\frac{b c \Gamma(k+l+c)}{[a(m+1)]^{k+l+c}}\right]
$$

\subsection{Stress-strength parameter}

Suppose that $X$ and $Y$ are two independent MGLFR RVs with respective parameters $\left(a, b, c, \theta_{1}\right)$ and $\left(a, b, c, \theta_{2}\right)$ having PDFs $f_{X}($.$) and f_{Y}($.$) , respectively. Let X$ be the strength of a system, which is subjected to stress $\mathrm{Y}$; therefore the stress-strength measures the system performance. Then stressstrength parameter, i.e. $R=P(Y<X)$, is as follows,

$$
R=P(Y<X)=\int_{0}^{\infty} P(Y<X \mid Y=y) f_{Y}(y) d y=\int_{0}^{\infty}\left(1-F_{X}\left(y, a, b, c, \theta_{1}\right)\right) f_{Y}\left(y, a, b, c, \theta_{2}\right) d y=\frac{\theta_{1}}{\theta_{1}+\theta_{2}}
$$

\subsection{Reliability function}

If $X$ has $M G L F R D(a, b, c, \theta)$, then the reliability function at time $t$ is given as follows:

$S(t, a, b, c, \theta)=1-\left[1-\exp \left(-\left(a t+b t^{c}\right)\right)\right]^{\theta}, t>0, a \geq 0, c>0, b \geq 0, \theta>0$.

This shows that increasing $\theta$ results upper reliability. If $\theta=1$ then $S(t)=\exp \left(-\left(a t+b t^{c}\right)\right)$, the higher values of $a$ and $b$ result less reliability.

\subsection{Hazard function}

If $X$ has $M G L F R D(a, b, c, \theta)$, then the hazard function at time $t$, is given as follows:

$$
h(t)=\frac{f(t)}{S(t)}=\frac{\theta\left(a+b c t^{c-1}\right)\left[1-\exp \left(-\left(a t+b t^{c}\right)\right)\right]^{\theta-1} \exp \left(-\left(a t+b t^{c}\right)\right)}{1-\left[1-\exp \left(-\left(a t+b t^{c}\right)\right)\right]^{\theta}},
$$

if $\theta=1$ then $h(t)=a+b c t^{c-1}$. In addition, if $c=1$, then the hazard function is a constant function, if $c=2$, then the hazard function is an ascending line, If $c=3$, then the hazard function is ascending curve. The reversed hazard function of $\operatorname{MG} \operatorname{LFR}(a, b, c, \theta)$ is: 


$$
\begin{aligned}
r(t, a, b, c, \theta) & =\frac{f(t, a, b, c, \theta)}{F(t, a, b, c, \theta)}=\frac{\theta\left(a+b c t^{c-1}\right)\left[1-\exp \left(-\left(a t+b t^{c}\right)\right)\right]^{\theta-1} \exp \left(-\left(a t+b t^{c}\right)\right)}{\left[1-\exp \left(-\left(a t+b t^{c}\right)\right)\right]^{\theta}} \\
& =\frac{\theta\left(a+b c t^{c-1}\right) \exp \left(-\left(a t+b t^{c}\right)\right)}{\left[1-\exp \left(-\left(a t+b t^{c}\right)\right)\right]}=\theta \frac{f(t, a, b, c, 1)}{F(t, a, b, c, 1)}=\theta r(t, a, b, c, 1)
\end{aligned}
$$

\section{Fuzzy reliability function}

Sometimes we are faced with situations that the parameters of lifetime variable cannot be expressed as crisp values. They can be stated as "approximately", "around", "between", or "about". Fuzzy sets theory is a useful tool for conveying these expressions into mathematical functions. In this case, reliability theory should be considered with respect to fuzzy rules. Buckley (2006) analyzed probability density functions when their parameters are fuzzy. We may consider the MGLFR distribution with fuzzy parameters and trapezoidal fuzzy number of $\tilde{a}$ that is replaced instead of $a$ in MGLFR distribution. In this case, we show the fuzzy probability of obtaining a value in the interval $\left[c^{*}, d^{*}\right], c^{*} \geq 0$ is as $\tilde{P}\left(c^{*} \leq X \leq d^{*}\right)$ and compute its $\alpha$-cut as follows: ( For more details, refer to Buckley, 2006)

$$
\tilde{P}\left(c^{*} \leq X \leq d^{*}\right)[\alpha]=\left\{\int_{c^{*}}^{d^{*}} f(x, a, b, c, \theta) d x \mid a \in \tilde{a}[\alpha]\right\}=\left[P^{L}[\alpha], P^{U}[\alpha]\right]
$$

where

$$
P^{L}[\alpha]=\min \left\{\int_{c^{*}}^{d^{*}} f(x, a, b, c, \theta) d x \mid a \in \tilde{a}[\alpha]\right\}, P^{U}[\alpha]=\max \left\{\int_{c^{*}}^{d^{*}} f(x, a, b, c, \theta) d x \mid a \in \tilde{a}[\alpha]\right\} .
$$

We represent parameter $\tilde{a}$ with a trapezoidal fuzzy number as $\tilde{a}=\left(a_{1}, a_{2}, a_{3}, a_{4}\right)$ such that we can describe a membership function $\xi_{\tilde{a}}(x)$ in the following manner:

$$
\xi_{\tilde{a}}(x)= \begin{cases}\frac{x-a_{1}}{a_{2}-a_{1}}, & a_{1} \leq x<a_{2} \\ 1, & a_{2} \leq x \leq a_{3} \\ \frac{a_{4}-x}{a_{4}-a_{3}}, & a_{3}<x \leq a_{4} \\ 0, & \text { not }\end{cases}
$$

The $\alpha$-cut $\tilde{a}$ denote as $\tilde{a}[\alpha]=\left[a_{1}+\left(a_{2}-a_{1}\right) \alpha, a_{4}-\left(a_{4}-a_{3}\right) \alpha\right]$

Fuzzy reliability (or fuzzy survival) function $(\tilde{S}(t))$ is the fuzzy probability a unit survives beyond time $t$. Let the random variable $X$ denote lifetime of a system component, also let $\mathrm{X}$ follow fuzzy density function $f(x, \tilde{\theta})$ and fuzzy cumulative distribution function $\tilde{F}_{X}(t)=\tilde{P}(X \leq t)$ where parameter $\tilde{\theta}$ is a fuzzy number, in these conditions the fuzzy reliability function at time $t$ is defined as (See Baloui Jamkhaneh, 2014):

$$
\tilde{S}(t)[\alpha]=\tilde{P}(X>t)[\alpha]=\left\{\int_{t}^{\infty} f(x, \theta) d x \mid \theta \in \tilde{\theta}[\alpha]\right\}=\left[S^{L}(t)[\alpha], S^{U}(t)[\alpha]\right], t>0,
$$

where

$$
S^{L}(t)[\alpha]=\min \left\{\int_{t}^{\infty} f(x, \theta) d x \mid \theta \in \tilde{\theta}[\alpha]\right\} \quad \text { and } S^{U}(t)[\alpha]=\max \left\{\int_{t}^{\infty} f(x, \theta) d x \mid \theta \in \tilde{\theta}[\alpha]\right\}
$$

Therefore, fuzzy reliability function based on fuzzy MGLFRD distribution is as follows: 
$\tilde{S}(t, b, c, \theta)[\alpha]=\left\{\int_{t}^{\infty} \theta\left(a+b c x^{c-1}\right)\left[1-\exp \left(-\left(a x+b x^{c}\right)\right)\right]^{\theta-1} \exp \left(-\left(a x+b x^{c}\right)\right) d x \mid a \in \tilde{a}[\alpha]\right\}$,

$\tilde{S}(t, b, c, \theta)[\alpha]=\left\{1-\left[1-\exp \left(-\left(a t+b t^{c}\right)\right)\right]^{\theta} \mid a \in \tilde{a}[\alpha]\right\}, t>0, c>0, b \geq 0, \theta>0$.

According to the function of $1-\left[1-\exp \left(-\left(a t+b t^{c}\right)\right)\right]^{\theta}$ that is decreasing in terms of $a$, we have:

$\tilde{S}(t, b, c, \theta)[\alpha]=\left[1-\left[1-\exp \left(-\left(\left(a_{4}-\left(a_{4}-a_{3}\right) \alpha\right) t+b t^{c}\right)\right)\right]^{\theta}, 1-\left[1-\exp \left(-\left(\left(a_{1}+\left(a_{2}-a_{1}\right) \alpha\right) t+b t^{c}\right)\right)\right]^{\theta}\right.$

Upper and lower bound of $\tilde{S}(t, b, c, \theta)[\alpha]$ are two dimensional functions in terms of $\alpha$ and $t($ $0 \leq \alpha \leq 1$ and $t>0)$. For any particular value of $t_{0}, \tilde{S}\left(t_{0}, b, c, \theta\right)[\alpha]$ is a fuzzy number. Finally, $\tilde{S}\left(t_{0}, b, c, \theta\right)\left[\alpha_{0}\right]=\left[S^{L}\left(t_{0}\right)\left[\alpha_{0}\right], S^{U}\left(t_{0}\right)\left[\alpha_{0}\right]\right]$ is $\alpha_{0}$-cut of fuzzy reliability of a unit. In this method, for any particular level of $\alpha_{0}$, upper and lower bound of $\tilde{S}(t, b, c, \theta)\left[\alpha_{0}\right]$ are two functions in terms of $t$. So, in this case reliability curve is like a band with upper and lower bound whose width depends on the ambiguity parameter (See Baloui Jamkhaneh, 2011). Fig. 1 shows $\alpha$-cut of fuzzy reliability with $\alpha=$ 0 and 1. Fig. 2 shows that by increasing of the value of $c$, when $t$ is small, we may find higher reliability band and for large value of $t$, we may find lower reliability band.

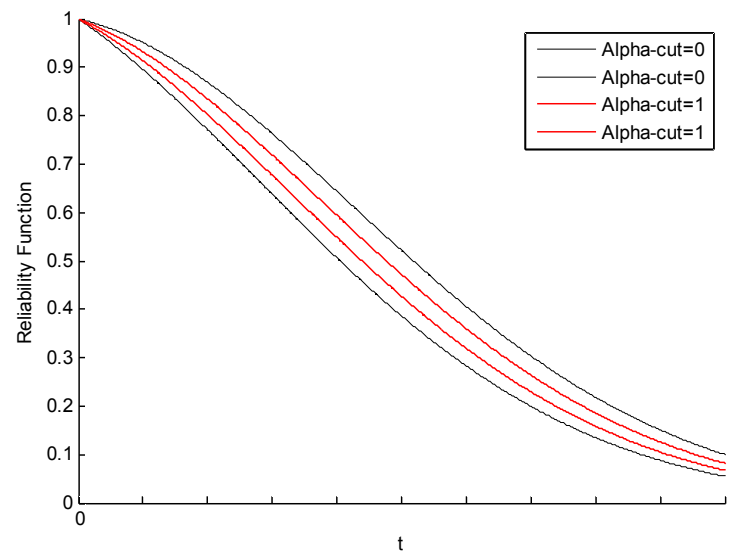

Fig. 1. $\alpha$-cut of fuzzy reliability for different $t$

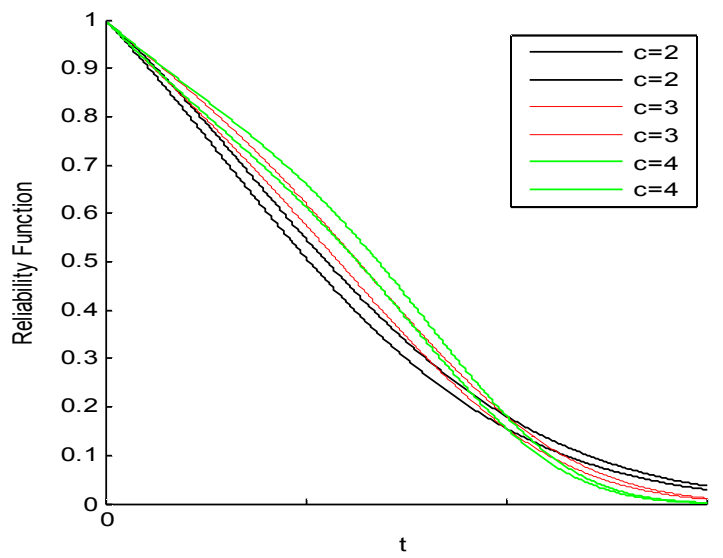

Fig. 2. $\alpha$-cut of fuzzy reliability for different $c$

Fig. 3 shows the behavior of reliability band $\tilde{S}(t)[0]$ with $c=b=2$ under various conditions. This figure shows that decreasing $\theta$ results lower reliability band, which means the reliability will be lower under these conditions.

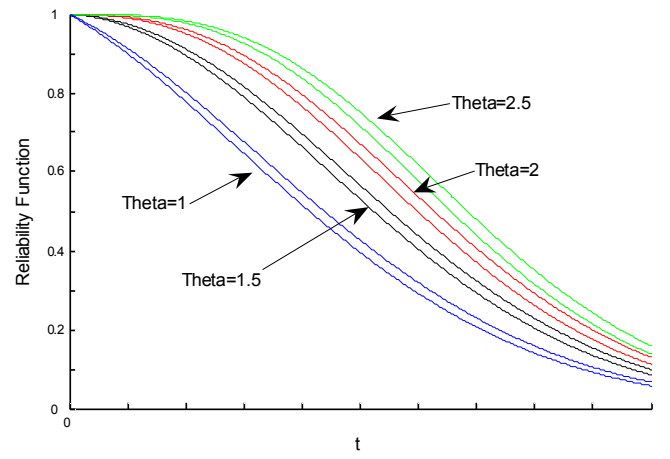

Fig. 3. $\alpha$-cut of fuzzy survival ( $\alpha=0$ ) for different $\theta$

If $\theta=1$ then fuzzy reliability fuzzy is as follows, 
$\tilde{S}(t)[\alpha]=\left[\exp \left(-\left(\left(a_{4}-\left(a_{4}-a_{3}\right) \alpha\right) t+b t^{c}\right)\right), \exp \left(-\left(\left(a_{1}+\left(a_{2}-a_{1}\right) \alpha\right) t+b t^{c}\right)\right)\right]$

For $t_{0}$, reliability function is a fuzzy number and membership function of $\tilde{S}\left(t_{0}\right)$ is as follows,

$$
\xi_{\tilde{S}\left(t_{0}\right)}(x)=\left\{\begin{array}{cc}
\frac{x e^{b t_{0}^{c}}-e^{-a_{4} t_{0}}}{e^{-a_{3} t_{0}}-e^{-a_{4} t_{0}}}, & \left.\exp \left\{-a_{4} t_{0}-b t_{0}^{c}\right)\right\} \leq x<\exp \left\{-a_{3} t_{0}-b t_{0}^{c}\right\} \\
1 \quad & \left.\exp \left\{-a_{3} t_{0}-b t_{0}^{c}\right)\right\} \leq x<\exp \left\{-a_{2} t_{0}-b t_{0}^{c}\right\} \\
\frac{e^{-a_{1} t_{0}}-x e^{b t_{0}^{c}}}{e^{-a_{1} t_{0}}-e^{-a_{2} t_{0}}}, & \left.\exp \left\{-a_{2} t_{0}-b t_{0}^{c}\right)\right\} \leq x<\exp \left\{-a_{1} t_{0}-b t_{0}^{c}\right\}
\end{array}\right.
$$

for every $b, c$, the value of $e^{b t_{0}^{c}}$ is greater than or equal to 1 and $e^{b t_{0}^{c}}$ is a non-decreasing function of $b$. If $b=0$, then fuzzy number of reliability will have its maximum value with the lowest uncertainty. As the values of $b$ increases, we get lower values for fuzzy numbers of reliability with more uncertainty.

\section{Fuzzy hazard function}

Another fuzzy characterizes of the lifetime distribution is the fuzzy hazard function $\tilde{h}(t)$. This function is also known as the instantaneous failure rate function. We propose the concept of a fuzzy hazard function based on the fuzzy probability measure and named $\alpha$-cut hazard band. The fuzzy hazard function $\tilde{h}(t)$ is the fuzzy conditional probability of an item failing in the short time interval $t$ to $(t+d t)$ given that it has not failed at time $t$. Mathematically, we would define the fuzzy hazard function as

$$
\begin{aligned}
\tilde{h}(t)[\alpha] & =\lim _{\Delta t \rightarrow 0} \frac{\tilde{P}(t<X<t+\Delta t \mid X>t)}{\Delta t}=\left\{\lim _{\Delta t \rightarrow 0} \frac{S(t)-S(t+\Delta t)}{\Delta t S(t)} \mid a \in \tilde{a}[\alpha]\right\} \\
& =\left\{\frac{-S^{\prime}(t)}{S(t)} \mid a \in \tilde{a}[\alpha]\right\}=\left\{\frac{f(t)}{S(t)} \mid a \in \tilde{a}[\alpha]\right\} .
\end{aligned}
$$

The fuzzy MGLFRD distribution has the following fuzzy hazard function,

$$
h(t)[\alpha]==\left\{\frac{\theta\left(a+b c t^{c-1}\right)\left[1-\exp \left(-\left(a t+b t^{c}\right)\right)\right]^{\theta-1} \exp \left(-\left(a t+b t^{c}\right)\right)}{1-\left[1-\exp \left(-\left(a t+b t^{c}\right)\right)\right]^{\theta}} \mid a \in \tilde{a}[\alpha]\right\},
$$

If $\theta=1$ then

$$
h(t)[\alpha]=\left\{a+b c t^{c-1} \mid a \in \tilde{a}[\alpha]\right\},
$$

and $\alpha$-cut of $\tilde{h}(t)$ is as follows,

$$
h(t)[\alpha]=\left[a_{1}+\left(a_{2}-a_{1}\right) \alpha+b c t^{c-1}, a_{4}-\left(a_{4}-a_{3}\right) \alpha+b c t^{c-1}\right]
$$

i) If $c=1$, then the hazard function is a fuzzy number (see Fig. 4) 


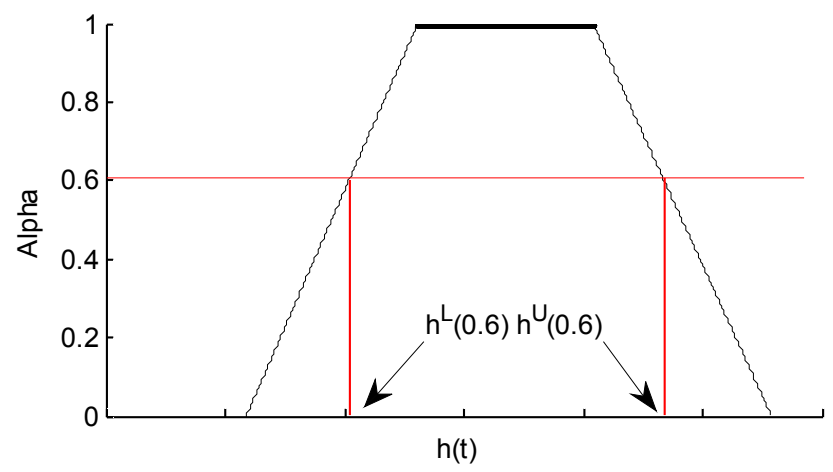

Fig. 4. membership function and $\alpha$-cut ( $\alpha=0.6)$ of fuzzy hazard $(c=1)$

ii) If $c=2$, then for every $\alpha$-cut, the hazard band is ascending with linear upper and lower bounds.

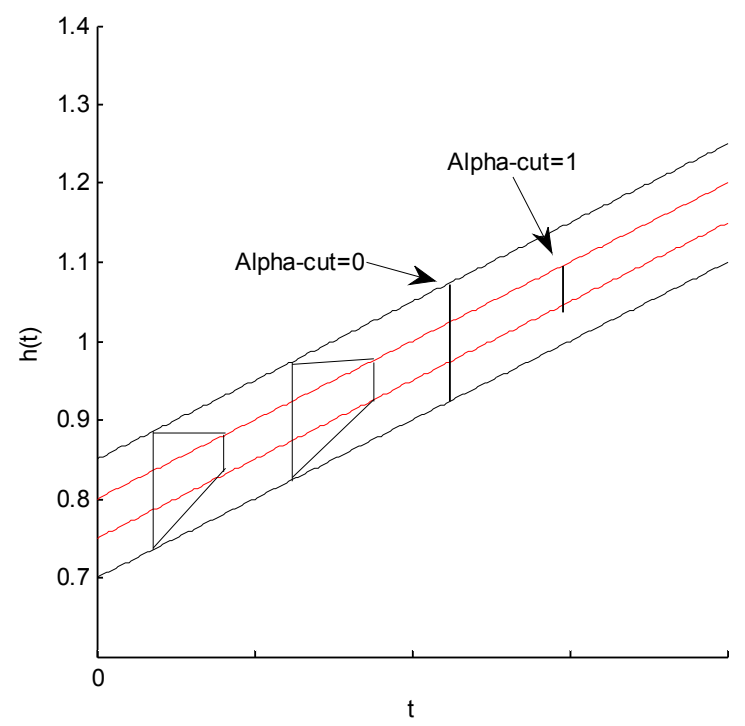

Fig. 5. $\alpha$-cut $(\alpha=0,1)$ of fuzzy hazard $(c=2)$

iii) If $c=3$, then for every $\alpha$-cut, the hazard band is ascending with curve trend behavior in upper and lower bound.

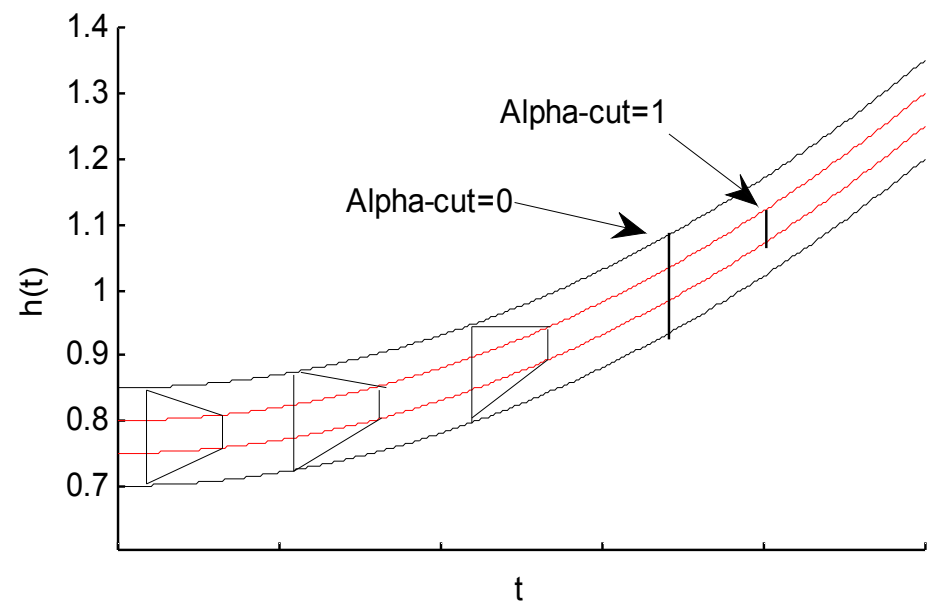

Fig. 6. $\alpha-\operatorname{cut}(\alpha=0,1)$ of fuzzy hazard $(c=3)$ 


\section{Conclusions}

In this paper, we have introduced a new four-parameter modified generalized linear failure rate distribution and different properties of the new model have been presented. It is observed that the proposed MGLFR distribution has several desirable properties and several existing well known distributions can be obtained as special cases of this distribution. It is observed that the MGLFR distribution can have non-decreasing, non-increasing and bathtub shaped hazard rate functions, which are quite desirable for data analysis purposes. The fuzzy probability theory has been successfully applied to the reliability system in this paper. Whenever, the lifetimes of components and parameters follow randomness and fuzziness, respectively, conventional reliability system is not feasible. Thus, we have applied successfully the fuzzy distribution to overcome this difficulty.

\section{Acknowledgement}

The author would like to thank the anonymous referees for constructive comments on earlier version of this paper.

\section{References}

Aliev, I. M., \& Kara, Z. (2004). Fuzzy system reliability analysis using dependent fuzzy set. Control and Cybernetics, 33, 653-662.

Baloui Jamkhaneh, E. (2011). An Evaluation of the Systems Reliability Using Fuzzy Lifetime Distribution. Journal of Applied Mathematics, Islamic Azad University of Lahijan, 8(28), 73-80.

Baloui Jamkhaneh. E., \& Nozari, A. (2012). Fuzzy System Reliability Analysis Based on Confidence Interval. Advanced Materials Research, 433, 4908-4914.

Baloui Jamkhaneh, E. (2014). Analyzing system reliability using fuzzy Weibull lifetime distribution. International Journal of Applied Operational Research, 4(1), 81-90.

Buckley, J.J. (2006). Fuzzy probability and statistics. Springer-Verlage Berlin Heidelberg.

Burr, I.W. (1942). Cumulative frequency distribution. Annals of Mathematical Statistics, 13, 215-232.

Cai, K.Y., Wen, C.Y. \& Zhang, M.L. (1991). Fuzzy variables as a basic for a theory of fuzzy reliability in the possibility context. Fuzzy Set and Systems, 42, 145-172.

Cai, K.Y., Wen, C.Y. \& Zhang, M.L. (1993). Fuzzy states as for a theory of fuzzy reliability. Microelectron Reliability, 33, 2253-2263.

Cai, K.Y. (1996). System failure engineering and fuzzy methodology. An introductory overview. Fuzzy Set and Systems, 83, 113-133.

Chen, C.H., Mon, L. (1993). Fuzzy system reliability analysis by interval of confidence. Fuzzy Set and Systems, 56, 29-35.

Garg, H., Rani, M., \& Sharma. S.P. (2013). Reliability analysis of the engineering systems using intuitionistic fuzzy set theory. Journal of Quality and Reliability Engineering, Article ID 943972, 10 pages.

Guo, R., Zhao R.Q., \& Li, X. (2007). Reliability analysis based on scalar fuzzy variables. Economic Quality Control, 22(1), $55-70$.

Guo, R., Zhao, R.Q., Guo, D., \& Dunne T. (2007). Random fuzzy variable modeling on repairable system. Journal of Uncertain Systems, 1(3), 222-234.

Gupta, R.D., Kundu D. (1999a). Generalized exponential distributions. Australian and New Zealand Journal of Statistics, 41(2), 173 - 188.

Karpisek, Z., Stepanek, P., \& Jurak, P. (2010). Weibull fuzzy probability distribution for reliability of concrete structures. Engineering Mechanics, 17(5/6), 363-372.

Mudholkar, G.S., Srivastava, D.K. (1993). Exponentiated Weibull family for analyzing bathtub failure rate data. IEEE Transactions on Reliability, 42, 299-302. 
Pak, A., Parham Gh. A., \& Saraj, M. (2013). Reliability estimation in Rayleigh distribution based on fuzzy lifetime data. International Journal of System Assurance Engineering and Management, published online August 2013.

Sarhan, A., \& Kundu, D. (2009). Generalized linear failure rate distribution. Communications in Statistics - Theory and Methods, 38, 642 - 660.

Sarhan, A., \& Zaindin, M. (2009). Modified Weibull distribution. Applied Sciences, 11, 123-136.

Surles, J.G., \& Padgett, W.J. (2001). Inference for reliability and stress-strength for a scaled Burr Type $\mathrm{X}$ distribution. Lifetime Data Analysis, 7, 187-200.

Hammer, M. (2001). Application of fuzzy theory to electrical machine reliability.Zeszyty Naukowe. Elektryka/Politechnika Śląska, 177, 161-166.

Onisawa, T., \& Kacprzyk, J. (1995). Reliability and safety analysis under fuzziness. Physica-Verlag Heidelberg.

Utkin, L. V. (1994). Knowledge based fuzzy reliability assessment. Microelectronics Reliability, 34(5), 863-874.

Utkin, L.V. (1994). Fuzzy reliability of repairable systems in the possibility context. Microelectron. Reliability, 34, 1865-1876.

Utkin, L.V., \& Gurov, S.V. (1995). A general formal approach for fuzzy reliability analysis in the possibility context. Fuzzy Set and Systems, 83, 203-213.

Wu, H.-C. (2006). Fuzzy Bayesian system reliability assessment based on exponential distribution. Applied Mathematics Modeling, 30, 509-530.

Yao, J.S., Su, J.S., \& Shih, T.S. (2008). Fuzzy system reliability analysis using triangular fuzzy numbers based on statistical data. Journal of information science and engineering, 24, 1521-1535. 